\title{
Normal Mode Spectrum of the Parallel-Chain $\beta$-Sheet*
}

\author{
JAGDEESH BANDEKAR and S. KRIMM, Biophysics Research \\ Division and Department of Physics, University of Michigan, \\ Ann Arbor, Michigan 48109
}

\begin{abstract}
Synopsis
Normal mode calculations have been carried out for parallel-chain $\beta$-sheet structures. These include the parallel-chain pleated sheet of poly(L-alanine) and the parallel-chain rippled sheet of polyglycine. Dipole derivative coupling has been included for amide I and II modes, and the effects of parallel-sheet and antiparallel-sheet arrangements of varying separation have been examined for the poly(L-alanine) case. Some amide and nonamide modes are distinctly different from their antiparallel-chain counterparts, thus providing a basis for distinguishing between such structures from their ir and Raman spectra. As in our previous studies, these results emphasize the need for both kinds of spectral data in order to draw definitive conclusions about conformation.
\end{abstract}

\section{INTRODUCTION}

The ir and Raman spectra of a protein derive from the detailed threedimensional structure of the molecule. While it is difficult to extract complete structural information directly from the vibrational spectra of such complex molecules, we have shown ${ }^{1}$ that normal mode analysis can provide a complete understanding of the spectra of known secondary structural components of proteins, such as the $\alpha$-helix, $\beta$-sheet, and $\beta$ - and $\gamma$-turns. These studies have helped in the interpretation of hitherto unassignable bands in the spectra of proteins, ${ }^{1}$ and are expected to lead eventually to the possibility of detailed analyses for protein molecules themselves. ${ }^{2}$

In this paper, we extend the vibrational analysis previously done for the antiparallel-chain $\beta$-sheet ${ }^{3,4}$ to parallel-chain structures. As originally proposed, ${ }^{5,6}$ the $\beta$-sheet can occur with adjacent chains in the hydrogen-bonded sheet parallel or antiparallel to each other. The antiparallel-chain structures, whether rippled ${ }^{7,8}$ or pleated, ${ }^{9}$ have been well characterized by diffraction studies, and detailed normal mode analyses of rippled-sheet polyglycine $\mathrm{I}^{10}$ and pleated-sheet $\beta$-poly(L-alanine) $)^{3,4}$ have been achieved. Comparable analyses of parallel-chain $\beta$-sheet structures have not been done, primarily because of the absence of well-defined model systems. However, crystal structure studies reveal that parallel-chain $\beta$-sheet conformations can be an important structural component of globular proteins: lactate dehydrogenase contains a six-stranded parallel-chain $\beta$-sheet ${ }^{11}$; triose phosphate isomerase has a parallel-chain $\beta$-sheet wrapped into a cylinder ${ }^{12}$ (a so-called $\beta$-barrel

\footnotetext{
*This is paper number 39 in a series on "Vibrational Analysis of Peptides, Polypeptides, and Proteins," of which paper number 38 is Ref. 19.
} 
conformation); carboxypeptidase ${ }^{13}$ and subtilisin $\mathrm{BPN}^{14}$ have a substantial proportion of residues in parallel-chain $\beta$-sheet structures; and rhodanese has $15 \%$ of its residues in parallel-chain $\beta$-sheet conformations, with none in the antiparallel-chain conformation. ${ }^{15}$ It is therefore important to characterize the vibrational spectrum of the parallel-chain $\beta$-sheet structure.

Here we treat the case of the flat sheet; twisted sheets ${ }^{16}$ will be dealt with separately. Although there is at present no well-established model polypeptide structure for such flat sheets (some dipeptides do crystallize with this type of hydrogen bonding ${ }^{17}$ ), we believe our results on the normal mode analyses of the canonical structures ${ }^{5,6}$ are reliable, since such an analysis applied to the tripeptide L-valyl-glycyl-glycine (VGG), known from crystal structure studies to form a parallel-chain $\beta$-structure, ${ }^{18}$ gives excellent agreement with observed ir and Raman spectra. ${ }^{19}$ To the extent that real structures deviate from the canonical structures, the results presented here will need to be modified. However, they serve as important indicators of how the vibrational modes of the parallel-chain $\beta$-sheet differ from those of the antiparallel-chain structures.

\section{NORMAL :IODE CALCULATIONS}

The structures used in our calculations were those given by Pauling and Corey, ${ }^{5,6}$ based on model-building studies, for the parallel-chain pleated sheet (PPS) and the parallel-chain rippled sheet (PRS). The calculation for the PPS was done on a poly(L-alanine), while that for the PRS was done on a polyglycine (since this is the only polypeptide that can by itself satisfy the requirement ${ }^{5}$ that alternate chains in the sheet adopt all-L and all-D configurations ${ }^{7,8}$ ). The PPS and PRS structures are shown schematically in Fig. $1(a$ and $b)$, respectively.

Standard borid lengths and angles ${ }^{1}$ (including planar peptide groups) were used to generate chains having the proposed ${ }^{6}$ intrachain repeat of $b=6.50 \AA$ and interchain repeat of $a=4.85 \AA$ (PPS) or $a=9.60 \AA$ (PRS) (doubled in the latter case because adjacent chains are related to each other by a glide plane). The structural parameters of these two $\beta$-sheets are given in Table I, where they are compared with their counterparts for the comparable antiparallel-chain (APPS and APRS) $\beta$-sheets. The sheet repeat of the PPS contains two residues, related to each other by a twofold screw axis parallel to the chain axis $C_{2}^{S}(b)$; The modes for this $C_{2}$ group are distributed between two symmetry species, $\mathrm{A}$ and $\mathrm{B}$, which are both ir and Raman active. The repeat of the PRS contains four residues, related to each other by the same $\mathrm{C}_{2}^{\mathrm{s}}(b)$ axis and by glide planes parallel to the sheet, $\sigma_{\mathrm{v}}^{\mathrm{g}}(\mathrm{xz})$, and perpendicular to the sheet, $\sigma_{v}^{g}(y z)$. The modes for this $\mathrm{C}_{2 \mathrm{v}}$ group are distributed between four symmetry species- $A_{1}, A_{2}, B_{1}$, and $B_{2}$-with all modes being Raman active, while only $A_{1}, B_{1}$, and $B_{2}$ are ir active.

Although the full normal mode calculation was done on only one sheet, resonance coupling effects were computed for an array of $11 \times 11$ residues in one sheet and an array of 11 sheets. These sheets were arranged with respect to each other as follows: first, the chains were shifted by $a / 2$ (PPS) or $a / 4$ (PRS) in the plane of the sheet, consistent with arrangements found in APPS poly(L-alanine $)^{9}$ and $\mathrm{VGG}^{18}$; second, both parallel and antiparallel arrange- 


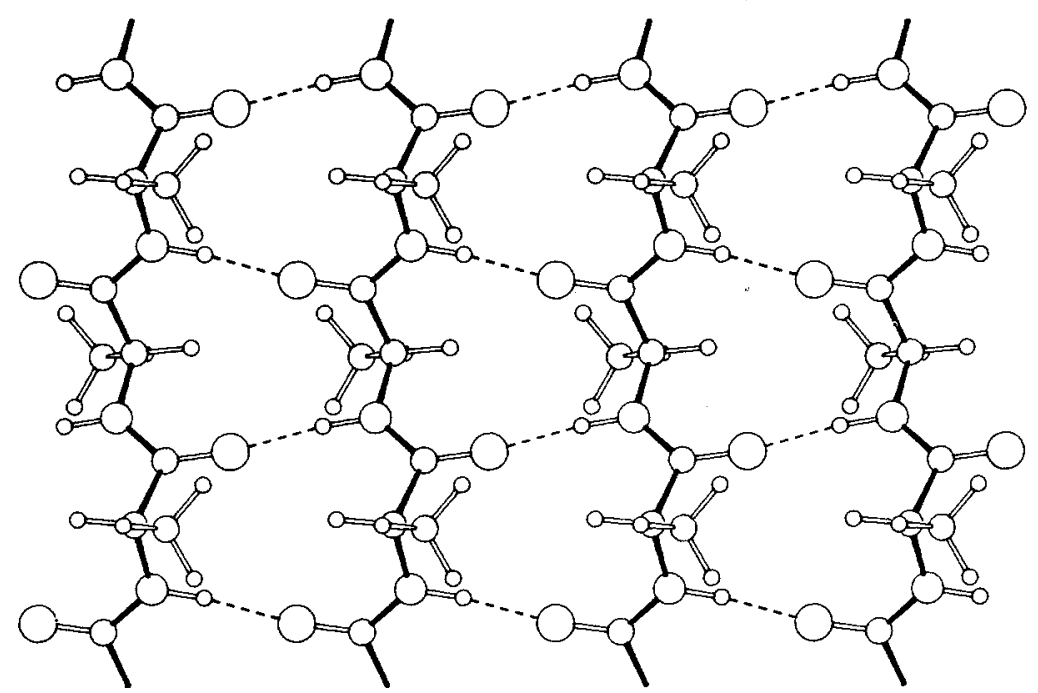

(a)

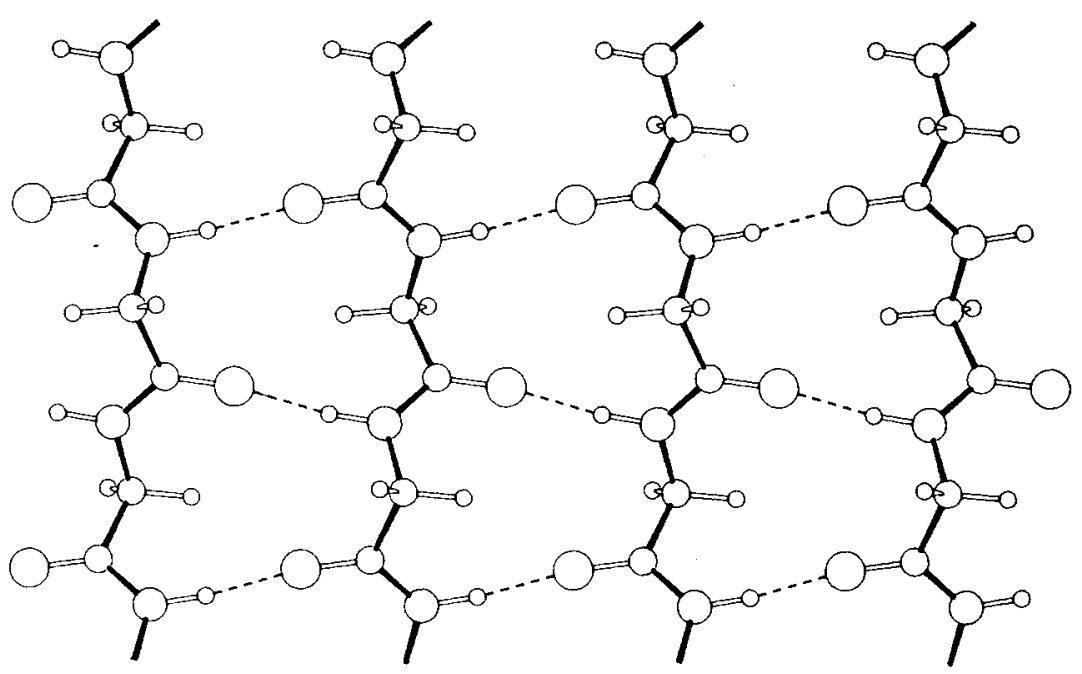

(b)

Fig. 1. (a) Parallel-chain pleated sheet of poly(L-alanine). (b) Parallel-chain rippled sheet of polyglycine.

ments of sheets were formed (in both cases with "in-phase" pleating of adjacent sheets, to maintain optimum packing of side chains); and third, the separation between sheets was set. This distance was varied from 5.0 to $17.0 \AA$ for the PPS, and set at $3.35 \AA$ for the PRS, the value for polyglycine $I^{7}$ (Resonance coupling calculations were also done for a $7 \times 7 \times 7$ array of the PPS. The results were the same for the parallel-sheet arrangement; for the antiparallel-sheet arrangement, the results were the same for the $B$ species, but the curve near $5 \AA$ sheet separation started at a value about $3 \mathrm{~cm}^{-1}$ lower.) 


\section{TABLE I}

Structural Parameters for Parallel-Chain Pleated (PPS) and Rippled (PRS) Sheet Structures and Their Antiparallel-Chain Counterparts

\begin{tabular}{rcccc}
\hline & PPS & APPS & PRS & APRS \\
\hline$a$ or $a / 2(\AA)$ & 4.85 & 4.73 & 4.80 & 4.77 \\
$b / 2(\AA)$ & 3.25 & 3.445 & 3.25 & 3.552 \\
$\phi\left({ }^{\circ}\right)$ & -119.0 & -138.4 & -119.0 & -149.9 \\
$\psi\left({ }^{\circ}\right)$ & 114.0 & 135.7 & 114.0 & 146.5 \\
$1(\mathrm{~N} \ldots$ O) $(\AA)$ & 2.74 & 2.73 & 2.82 & 2.91 \\
$1(\mathrm{H} \ldots \mathrm{O})(\AA)$ & 1.82 & 1.75 & 1.75 & 2.12 \\
$<\mathrm{HNO}\left({ }^{\circ}\right)$ & 5.5 & 9.8 & 4.9 & 31.4 \\
$<\mathrm{NHO}\left({ }^{\circ}\right)$ & 172.0 & 164.6 & 172.0 & 134.4 \\
\hline
\end{tabular}

The force field used for the PPS poly(L-alanine) was that refined for APPS poly(L-alanine), ${ }^{3,4}$ while that used for PRS polyglycine was the polyglycine I force field. ${ }^{10}$ While complete transferability in these cases is not strictly correct, since there are differences in hydrogen-bond geometry (see Table I), we believe the good agreement obtained when a similar procedure was used for $\mathrm{VGG}^{19}$ indicates that this should be a good first approximation. Resonance coupling interactions were computed for amide I and amide II modes by the dipole derivative coupling (DDC) method, ${ }^{20}$ as in $\mathrm{VGG},{ }^{19}$ using the dipole derivatives that we obtained from ab initio studies of the peptide group. ${ }^{21}$

\section{RESULTS AND DISCUSSION}

\section{Parallel-Chain Pleated Sheet}

The unperturbed normal mode frequencies and potential energy distributions (PED) for a single sheet of PPS poly(L-alanine) are given in Table II. The unperturbed frequencies and PEDs for an $\mathrm{N}$-deuterated sheet are given in Table III. In Table IV we collect the frequencies of the main amide modes, with the effects of DDC included for amide I and amide II. The effects of sheet separation on the DDC shifts for amide I are given in Fig. 2(a and b) for the parallel and antiparallel arrangements of sheets, respectively, and for amide II in Fig. 3( $a$ and $b)$ for the comparable structures.

The amide I modes of a single sheet are predicted at 1663(A) and 1642(B) $\mathrm{cm}^{-1}$. Symmetry considerations indicate that the A-species mode should be strong in the Raman and the B species mode strong in the ir. It is interesting to note that in VGG the comparable strong bands are found (and predicted) at $1657 \mathrm{VS}$ (1654) $\mathrm{cm}^{-1}$ in the Raman and 1645VS (1644) $\mathrm{cm}^{-1}$ in the ir. ${ }^{19}$ [An earlier calculation, ${ }^{22}$ based on a transition dipole moment derived from interactions within only one sheet, gave frequencies of 1660(A) and 1653(B) $\mathrm{cm}^{-1}$. If we use a transition dipole moment derived from the actual multisheet interaction in $\beta$-poly(L-alanine), ${ }^{23}$ we obtain frequencies of $1666(\mathrm{~A})$ and $1654 \mathrm{~cm}^{-1}$. A calculation based on resonance coupling within only one sheet and an empirically assigned unperturbed frequency ${ }^{24}$ gave values of 1651(A) and $1637(\mathrm{~B}) \mathrm{cm}^{-1}$.] 
TABLE II

Normal Modes of a Parallel-Chain Pleated Sheet of Poly(L-Alanine)

\begin{tabular}{|c|c|c|}
\hline \multicolumn{2}{|c|}{ Species } & \multirow[b]{2}{*}{ Potential Energy Distribution ${ }^{\mathbf{a}}$} \\
\hline A & $\mathbf{B}$ & \\
\hline 3244 & 3244 & $\mathrm{NH} s(97)$ \\
\hline 2984 & 2984 & $\mathrm{CH}_{3}$ as $2(56) \mathrm{CH}_{3}$ asl(44) \\
\hline 2983 & 2983 & $\mathrm{CH}_{3}$ as1(56) $\mathrm{CH}_{3}$ as2(44) \\
\hline 2929 & 2929 & $\mathrm{CH}_{3} \mathrm{ss}(100)$ \\
\hline 2866 & 2866 & $\mathrm{C}^{\alpha} \mathrm{H}^{\alpha} \mathrm{s}(99)$ \\
\hline 1678 & 1675 & $\mathrm{COs}(74) \mathrm{CN} s(20)$ \\
\hline 1574 & 1568 & NH ib(55) CN s(18) $\mathrm{C}^{\alpha} \mathrm{C} \mathrm{s}(10)$ \\
\hline \multirow[t]{2}{*}{1455} & & $\mathrm{CH}_{3} \mathrm{abl}(75) \mathrm{CH}_{3} \mathrm{rl}(11)$ \\
\hline & 1453 & $\mathrm{CH}_{3} \mathrm{ab} 2(85) \mathrm{CH}_{3} \mathrm{r} 2(10)$ \\
\hline \multirow[t]{2}{*}{1452} & & $\mathrm{CH}_{3} \mathrm{ab} 2(80)$ \\
\hline & 1451 & $\mathrm{CH}_{3} \mathrm{abl}(87) \mathrm{CH}_{3} \mathrm{r} 1(10)$ \\
\hline \multirow[t]{2}{*}{1399} & & $\mathrm{H}^{\alpha} \mathrm{b} 2(23) \mathrm{C}^{\alpha} \mathrm{Cs}(17) \mathrm{CH}_{3} \operatorname{sb}(17) \mathrm{H}^{\alpha} \mathrm{b} 1(11) \mathrm{NH} \mathrm{ib}(10)$ \\
\hline & 1384 & $\mathrm{CH}_{3} \mathrm{sb}(68) \mathrm{H}^{\alpha} \mathrm{b} 2(16) \mathrm{C}^{\alpha} \mathrm{C}^{\beta} \mathrm{s}(12)$ \\
\hline \multirow[t]{3}{*}{1371} & & $\mathrm{CH}_{3} \mathrm{sb}(66) \mathrm{H}^{\alpha} \mathrm{bl}(20)$ \\
\hline & 1330 & $\mathrm{NH} \mathrm{ib}(27) \mathrm{C}^{\alpha} \mathrm{Cs}(16) \mathrm{CH}_{3} \mathrm{sb}(16) \mathrm{H}^{\alpha} \mathrm{bl}(12) \mathrm{H}^{\alpha} \mathrm{b} 2(11)$ \\
\hline & 1303 & $\mathrm{H}^{\alpha} \mathrm{bl}(40) \mathrm{CN} \mathrm{s}(14) \mathrm{CO} \mathrm{ib(11)}$ \\
\hline 1243 & & $\mathrm{H}^{\alpha}$ bl(28) $\mathrm{NC}^{\alpha} \mathrm{s}(20) \mathrm{CN} s(17) \mathrm{NH}$ ib(14) \\
\hline \multirow[t]{2}{*}{1207} & & $\mathrm{NC}^{\alpha} \mathrm{s}(25) \mathrm{H}^{\alpha}$ bl(19) $\mathrm{C}^{\alpha} \mathrm{C} \mathrm{s}(10) \mathrm{NH}$ ib(8) \\
\hline & 1193 & $\mathrm{C}^{\alpha} \mathrm{C}^{\beta} \mathrm{s}(34) \mathrm{NC}^{\alpha} \mathrm{s}(31) \mathrm{H}^{\alpha} \mathrm{b} 1(19) \mathrm{CH}_{3} \mathrm{r} 2(10)$ \\
\hline \multirow[t]{3}{*}{1153} & & $\mathrm{H}^{\alpha} \mathrm{b} 2(40) \mathrm{C}^{\alpha} \mathrm{C}^{\beta} \mathrm{s}(30) \mathrm{CH}_{3} \mathrm{sb}(14) \mathrm{H}^{\alpha} \mathrm{b} 1(13)$ \\
\hline & 1133 & $\mathrm{H}^{\alpha} \mathrm{b} 2(17) \mathrm{CH}_{3} \mathrm{rl}(17) \mathrm{CN} \mathrm{s}(16) \mathrm{H}^{\alpha}$ b1(14) $\mathrm{CH}_{3} \mathrm{r} 2(10) \mathrm{NH}$ ib(6) \\
\hline & 1097 & $\mathrm{CH}_{3} \mathrm{r} 2(40) \mathrm{C}^{a} \mathrm{C}^{\beta} \mathrm{s}(17) \mathrm{CH}_{3} \mathrm{r} 1(15)$ \\
\hline \multirow[t]{2}{*}{1094} & & $\mathrm{CH}_{3} \mathrm{r} 2(32) \mathrm{CH}_{3} \mathrm{r} 1(24) \mathrm{C}^{\alpha} \mathrm{C}^{\beta} \mathrm{s}(18)$ \\
\hline & 1071 & $\mathrm{H}^{\alpha} \mathrm{b} 2(30) \mathrm{CH}_{3} \mathrm{r} 1(30) \mathrm{C}^{\alpha} \mathrm{C}^{\beta} \mathrm{s}(18)$ \\
\hline 1062 & & $\mathrm{C}^{\alpha} \mathrm{C}^{\beta} \mathrm{s}(43) \mathrm{CH}_{3} \mathrm{rl}(25) \mathrm{H}^{\alpha} \mathrm{b} 2(17)$ \\
\hline 968 & & $\mathrm{NC}^{\alpha} \mathrm{s}(33) \mathrm{CH}_{3} \mathrm{r} 2(26) \mathrm{CH}_{3} \mathrm{rl}(16) \mathrm{C}^{\alpha} \mathrm{C} \mathrm{s}(12)$ \\
\hline \multirow[t]{4}{*}{913} & & $\mathrm{CN} \mathrm{s}(15) \mathrm{C}^{\alpha} \mathrm{C} \mathrm{s}(14) \mathrm{CNC}^{\alpha} \mathrm{d}(13) \mathrm{CO} \mathrm{s}(11) \mathrm{C}^{\alpha} \mathrm{CN} \mathrm{d}(10)$ \\
\hline & 907 & $\mathrm{NC}^{\alpha} \mathrm{s}(25) \mathrm{CH}_{3} \mathrm{r} 2(18) \mathrm{CN} s(17)$ \\
\hline & 853 & $\mathrm{C}^{\alpha} \mathrm{Cs}(29) \mathrm{NC}^{\alpha} \mathrm{s}(21) \mathrm{C}^{\alpha} \mathrm{C}^{\beta} \mathrm{s}(17)$ \\
\hline & 771 & $\mathrm{CO}$ ib $(20) \mathrm{C}^{\alpha} \mathrm{Cs}(19) \mathrm{NC}^{\alpha} \mathrm{C} \mathrm{d}(15) \mathrm{NC}^{\alpha} \mathrm{s}(10) \mathrm{CH}_{3} \mathrm{r} 1(10)$ \\
\hline \multirow[t]{2}{*}{712} & & $\mathrm{NH}$ ob(42) $\mathrm{CN} t(41) \mathrm{CO}$ ob(19) $\mathrm{NH} \mathrm{t}(15)$ \\
\hline & 706 & $\mathrm{CN} t(77) \mathrm{NH}$ ob(32) NH t(19) \\
\hline 661 & & $\mathrm{CO}$ ob(44) $\mathrm{CN} \mathrm{t(30)} \mathrm{C}^{\beta}$ bl(16) CO ib(15) \\
\hline \multirow[t]{4}{*}{643} & & $\mathrm{CO} \mathrm{ib(35)} \mathrm{CO} o b(15) \mathrm{CN} \mathrm{t}(14) \mathrm{C}^{\beta} \mathrm{b} 2(13) \mathrm{C}^{\alpha} \mathrm{C} \mathrm{s}(12)$ \\
\hline & 628 & $\mathrm{CO}$ ob(71) $\mathrm{NH}$ ob(22) $\mathrm{C}^{\beta}$ b2(12) $\mathrm{C}^{\alpha} \mathrm{CN} \mathrm{d}(10)$ \\
\hline & 569 & $\mathrm{C}^{\alpha} \mathrm{CN} d(38) \mathrm{CO}$ ob(17) \\
\hline & 437 & $\mathrm{C}^{\beta}$ b1(68) NH ob(9) \\
\hline 417 & & $\mathrm{C}^{\beta} \mathrm{b} 2(63) \mathrm{NC}^{\alpha} \mathrm{C} \mathrm{d}(17) \mathrm{NH}$ ob $(10)$ \\
\hline \multirow[t]{2}{*}{355} & & $\mathrm{C}^{\beta} \mathrm{b} 1(14) \mathrm{C}^{\beta} \mathrm{b} 2(13) \mathrm{C}^{\alpha} \mathrm{CN} \mathrm{d}(12) \mathrm{C}^{\alpha} \mathrm{Cs}(11)$ \\
\hline & 299 & $\mathrm{CO} i b(36) \mathrm{NC}^{\alpha} \mathrm{C} \mathrm{d}(23) \mathrm{C}^{\beta} \mathrm{b} 2(16) \mathrm{CNC}^{\alpha} \mathrm{d}(14)$ \\
\hline \multirow[t]{2}{*}{296} & & $\mathrm{NC}^{\alpha} \mathrm{C} d(39) \mathrm{C}^{\alpha} \mathrm{CN} \mathrm{d}(22)$ \\
\hline & 246 & $\mathrm{C}^{\beta} \mathrm{b} 2(52) \mathrm{C}^{\alpha} \mathrm{C}^{\beta} \mathrm{t}(10)$ \\
\hline \multirow[t]{2}{*}{244} & & $\mathrm{C}^{\alpha} \mathrm{C}^{\beta} \mathrm{t}(84)$ \\
\hline & 239 & $\mathrm{C}^{\alpha} \mathrm{C}^{\beta} \mathrm{t}(87)$ \\
\hline 187 & & $\mathrm{CNC}^{\alpha} \mathrm{d}(41) \mathrm{NH}$ ob(15) \\
\hline \multirow[t]{2}{*}{169} & & $\mathrm{C}^{\alpha} \mathrm{CN} d(32) \mathrm{CNC}^{\alpha} \mathrm{d}(22) \mathrm{NH}$ ob(12) \\
\hline & 121 & $\mathrm{CNC}^{\alpha} \mathrm{d}(32) \mathrm{NC}^{\alpha} \mathrm{C} \mathrm{d}(21) \mathrm{C}^{\alpha} \mathrm{CN} \mathrm{d}(20)$ \\
\hline \multirow[t]{2}{*}{113} & & $\mathrm{CN} t(21) \mathrm{C}^{\beta} \mathrm{bl}(14) \mathrm{CO} \mathrm{ob}(13) \mathrm{CO} t(13) \mathrm{NH} t(13) \mathrm{NH}$ ob(12) \\
\hline & 113 & $\operatorname{COt}(43) \mathrm{NH}$ ob(23) $\mathrm{CN} t(19) \mathrm{NH} \mathrm{t}(16) \mathrm{C}^{\alpha} \mathrm{C} t(12)$ \\
\hline 55 & & $\mathrm{H} \ldots \mathrm{Os}(27) \mathrm{NH} \ldots \mathrm{Ob}(20) \mathrm{NH} \mathrm{ob}(14) \mathrm{CO} t(12)$ \\
\hline
\end{tabular}

${ }^{\text {a }}$, Stretch; as, antisymmetric stretch; ss, symmetric stretch; b, angle bend; ab, antisymmetric bend; sb, symmetric bend; ib, in-plane angle bend; ob, out-of-plane angle bend; w, wag; tw, twist; r, rock; $t$, torsion; $d$, deformation. Only contributions of 10 or greater are included, except for $\mathrm{NH}$ modes, where contributions $>5$ are included. If differences in PED are $\leq 4$, modes are not listed separately and average PED is given. 
TABLE III

Normal Modes of an N-Deuterated Parallel-Chain Pleated Sheet of Poly(L-Alanine)

\begin{tabular}{|c|c|c|}
\hline \multicolumn{2}{|c|}{ Species } & \multirow[b]{2}{*}{ Potential Energy Distribution ${ }^{a}$} \\
\hline A & B & \\
\hline 2984 & 2984 & $\mathrm{CH}_{3}$ as2(56) $\mathrm{CH}_{3}$ as1(43) \\
\hline 2983 & 2983 & $\mathrm{CH}_{3}$ asl(56) $\mathrm{CH}_{3}$ as2(43) \\
\hline 2929 & 2929 & $\mathrm{CH}_{3} \mathrm{ss}(100)$ \\
\hline 2866 & 2866 & $\mathrm{C}^{\alpha} \mathrm{H}^{\alpha} \mathrm{s}(99)$ \\
\hline 2382 & 2382 & ND s $(96)$ \\
\hline 1675 & 1670 & $\mathrm{COs}(73) \mathrm{CN} \mathrm{s}(21)$ \\
\hline \multirow[t]{2}{*}{1483} & & $\mathrm{C}^{\alpha} \mathrm{Cs}(25) \mathrm{CN} s(24) \mathrm{CO} \mathrm{ib}(17) \mathrm{CO} s(11) \mathrm{ND} \mathrm{ib}(10)$ \\
\hline & 1482 & $\mathrm{C}^{\alpha} \mathrm{C} s(24) \mathrm{CN} s(19) \mathrm{CO} \mathrm{ib}(14) \mathrm{ND}$ ib(9) \\
\hline 1452 & & $\mathrm{CH}_{3}$ ab2(78) $\mathrm{CH}_{3}$ ab1(11) \\
\hline \multirow[t]{3}{*}{1451} & & $\mathrm{CH}_{3} \mathrm{ab} 1(73)$ \\
\hline & 1445 & $\mathrm{CH}_{3}$ ab1(51) $\mathrm{CH}_{3}$ ab2(24) \\
\hline & 1378 & $\mathrm{CH}_{3} \mathrm{sb}(80)$ \\
\hline 1376 & & $\mathrm{CH}_{3} \mathrm{sb}(80) \mathrm{H}^{\alpha} \mathrm{b} 2(10)$ \\
\hline \multirow[t]{3}{*}{1332} & & $\mathrm{H}^{\alpha} \mathrm{b} 1(59) \mathrm{H}^{\alpha}$ b2(14) ND ib(7) \\
\hline & 1304 & $\mathrm{H}^{\alpha} \mathrm{b} 1(47) \mathrm{H}^{\alpha} \mathrm{b} 2(16) \mathrm{CN} s(10)$ \\
\hline & 1213 & $\mathrm{C}^{\alpha} \mathrm{C}^{\beta} \mathrm{s}(34) \mathrm{H}^{\alpha} \mathrm{b} 1(26) \mathrm{H}^{\alpha} \mathrm{b} 2(15) \mathrm{NC}^{\alpha} \mathrm{s}(12) \mathrm{CH}_{3} \mathrm{sb}(11) \mathrm{ND} \mathrm{ib}(10)$ \\
\hline \multirow[t]{2}{*}{1207} & & $\mathrm{NC}^{\alpha} \mathrm{s}(36) \mathrm{CH}_{3} \mathrm{r} 2(16)$ \\
\hline & 1167 & $\mathrm{CH}_{3} \mathrm{r} 2(28) \mathrm{NC}^{\alpha} \mathrm{s}(17) \mathrm{CH}_{3} \mathrm{r} 1(15)$ \\
\hline 1153 & & $\mathrm{H}^{\alpha} \mathrm{b} 2(36) \mathrm{C}^{\alpha} \mathrm{C}^{\beta} \mathrm{s}(31) \mathrm{H}^{\alpha} \mathrm{b} 1(22) \mathrm{CH}_{3} \mathrm{sb}(15)$ \\
\hline \multirow[t]{3}{*}{1107} & & $\mathrm{CH}_{3} \mathrm{r} 2(29) \mathrm{CH}_{3} \mathrm{rl}(22) \mathrm{C}^{\alpha} \mathrm{C}^{\beta} \mathrm{s}(10)$ \\
\hline & 1100 & $\mathrm{CH}_{3} \mathrm{r} 2(29) \mathrm{CH}_{3} \mathrm{r} 1(26) \mathrm{H}^{\alpha} \mathrm{b} 2(12) \mathrm{H}^{\alpha} \mathrm{bl}(10)$ \\
\hline & 1072 & $\mathrm{H}^{\alpha} \mathrm{b} 2(29) \mathrm{CH}_{3} \mathrm{rl}(27) \mathrm{C}^{\alpha} \mathrm{C}^{\beta} \mathrm{s}(25)$ \\
\hline 1065 & & $\mathrm{C}^{\alpha} \mathrm{C}^{\beta} \mathrm{s}(52) \mathrm{H}^{\alpha} \mathrm{b} 2(24) \mathrm{CH}_{3} \mathrm{rl}(10)$ \\
\hline \multirow[t]{2}{*}{1014} & & 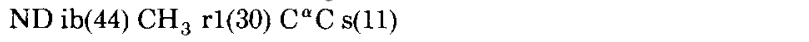 \\
\hline & 990 & ND ib(62) \\
\hline 946 & & $\mathrm{CH}_{3} \mathrm{r} 2(31) \mathrm{gC}^{\alpha} \mathrm{s}(30) \mathrm{ND} \mathrm{ib}(11)$ \\
\hline \multirow[t]{4}{*}{893} & & $\mathrm{CN} \mathrm{s}(20) \mathrm{CO} \mathrm{s}(13) \mathrm{C}^{\alpha} \mathrm{C} \mathrm{s}(12) \mathrm{CNC}^{\alpha} \mathrm{d}(12) \mathrm{C}^{\alpha} \mathrm{CN} \mathrm{d}(10)$ \\
\hline & 892 & $\mathrm{NC}^{\alpha} \mathrm{s}(30) \mathrm{CN} \mathrm{s}(17) \mathrm{CH}_{3} \mathrm{r} 2(17)$ \\
\hline & 850 & $\mathrm{C}^{\alpha} \mathrm{Cs}(30) \mathrm{NC}^{\alpha} \mathrm{s}(18) \mathrm{C}^{\alpha} \mathrm{C}^{\beta} \mathrm{s}(15) \mathrm{CN} \mathrm{s}(18)$ \\
\hline & 763 & $\mathrm{CO} i b(20) \mathrm{C}^{\alpha} \mathrm{Cs}(18) \mathrm{NC}^{\alpha} \mathrm{C} \mathrm{d}(13) \mathrm{NC}^{\alpha} \mathrm{s}(11) \mathrm{CNC}^{\alpha} \mathrm{d}(10)$ \\
\hline 678 & & $\mathrm{CO} \mathrm{ob}(73) \mathrm{C}^{\beta}$ bl(21) ND ob(13) \\
\hline \multirow[t]{4}{*}{644} & & $\mathrm{CO} \mathrm{ib(51)} \mathrm{C}^{\alpha} \mathrm{Cs}(15) \mathrm{C}^{\beta} \mathrm{b} 2(14) \mathrm{ND} \mathrm{ib}(6)$ \\
\hline & 630 & $\mathrm{CO}$ ob(77) $\mathrm{C}^{\beta}$ b2(9) ND ob(5) \\
\hline & 559 & $\mathrm{C}^{\alpha} \mathrm{CN}$ d(39) $\mathrm{CO}$ ob(14) \\
\hline & 512 & $\mathrm{CN} t(69) \mathrm{ND}$ ob(56) ND t(21) \\
\hline \multirow[t]{2}{*}{501} & & CN t(84) ND ob(34) ND t(19) \\
\hline & 430 & $\mathrm{C}^{\beta} \mathrm{bl}(62) \mathrm{CN} t(17)$ \\
\hline 412 & & $\mathrm{C}^{\beta} \mathrm{b} 2(60) \mathrm{NC}^{\alpha} \mathrm{C} d(16) \mathrm{ND}$ ob(11) \\
\hline \multirow[t]{2}{*}{353} & & $\mathrm{C}^{\beta} \mathrm{b} 2(17) \mathrm{C}^{\beta}$ b1(13) $\mathrm{C}^{\alpha} \mathrm{CN} \mathrm{d}(11) \mathrm{C}^{\alpha} \mathrm{C} \mathrm{s}(11)$ \\
\hline & 298 & $\mathrm{CO}$ ib(36) $\mathrm{NC}^{\alpha} \mathrm{C} d(23) \mathrm{C}^{\beta} \mathrm{b} 2(16) \mathrm{CNC}^{\alpha} \mathrm{d}(13)$ \\
\hline \multirow[t]{2}{*}{291} & & $\mathrm{NC}^{\alpha} \mathrm{C} d(39) \mathrm{C}^{\alpha} \mathrm{CN} \mathrm{d}(23)$ \\
\hline & 246 & $\mathrm{C}^{\beta} \mathrm{b} 2(52) \mathrm{C}^{\alpha} \mathrm{C}^{\beta} \mathrm{t}(10)$ \\
\hline \multirow[t]{2}{*}{244} & & $\mathrm{C}^{\alpha} \mathrm{C}^{\beta} \mathrm{t}(83)$ \\
\hline & 239 & $\mathrm{C}^{\alpha} \mathrm{C}^{\beta} \mathrm{t}(87)$ \\
\hline 185 & & $\mathrm{CNC}^{\alpha} \mathrm{d}(41) \mathrm{ND}$ ob(15) \\
\hline \multirow[t]{2}{*}{167} & & $\mathrm{C}^{\alpha} \mathrm{CN} \mathrm{d}(32) \mathrm{CNC}^{\alpha} \mathrm{d}(23) \mathrm{ND}$ ob(11) \\
\hline & 120 & $\mathrm{CNC}^{\alpha} \mathrm{d}(32) \mathrm{NC}^{\alpha} \mathrm{Cd}(21) \mathrm{C}^{\alpha} \mathrm{CN} \mathrm{d}(20)$ \\
\hline \multirow[t]{2}{*}{113} & & $\mathrm{CN} \mathrm{t}(21) \mathrm{C}^{\beta}$ bl(14) $\mathrm{CO}$ ob(13) $\mathrm{CO}$ t(13) ND t(13) ND ob(12) \\
\hline & 112 & $\mathrm{CO} \mathrm{t}(44) \mathrm{ND} o b(22) \mathrm{CN} \mathrm{t}(18) \mathrm{ND} t(17) \mathrm{C}^{\alpha} \mathrm{C} \mathrm{t}(12)$ \\
\hline 55 & & $\mathrm{O} \ldots \mathrm{D} \mathrm{s}(27) \mathrm{ND} \ldots \mathrm{O} \mathrm{b}(20) \mathrm{ND}$ ob(14) $\mathrm{CO} \mathrm{t}(12)$ \\
\hline
\end{tabular}

${ }^{\mathrm{a}} \mathrm{s}$, Stretch; as, antisymmetric stretch; ss, symmetric stretch; b, angle bend; ab, antisymmetric bend; sb, symmetric bend; ib, in-plane angle bend; ob, out-of-plane angle bend; $w$, wag; tw, twist; $\mathbf{r}$, rock; $t$, torsion; $d$, deformation. Only contributions of 10 or greater are included, except for ND modes, where contributions $>5$ are included. If differences in PED are $\leq 4$, modes are not listed separately and average PED is given. 
TABLE IV

Calculated Amide Frequencies (in $\mathrm{cm}^{-1}$ ) of Single-Sheet Parallel-Chain $\beta$-Structures

\begin{tabular}{ccl}
\hline Mode & PPS $^{\text {a }}$ Poly(L-Alanine) & PRS ${ }^{\mathrm{b}}$ Polyglycine \\
\hline I & A-1663 & $\mathrm{A}_{1}-1647$ \\
& B-1642 & $\mathrm{A}_{2}-1721$ \\
& & $\mathrm{~B}_{1}-1667$ \\
& & $\mathrm{~B}_{2}-1663$ \\
II & $\mathrm{A}-1553$ & \\
& $\mathrm{~B}-1586$ & $\mathrm{~A}_{1}-1526$ \\
& & $\mathrm{~A}_{2}-1535$ \\
& & $\mathrm{~B}_{1}-1560$ \\
III & $\mathrm{B}_{2}-1569$ \\
& $\mathrm{~A}-1399,1243$ & \\
& & $\mathrm{~A}_{1}-1405,1218$ \\
& & $\mathrm{~A}_{2}-1405,1219$ \\
& & $\mathrm{~B}_{1}-1290$ \\
V & $\mathrm{B}_{2}-1288$ \\
& $\mathrm{~A}-712$ & $\mathrm{~A}_{1}-698,680^{\mathrm{c}}$ \\
& $\mathrm{B}-706,628^{\mathrm{c}}$ & $\mathrm{A}_{2}-703,676^{\mathrm{c}}$ \\
& & $\mathrm{B}_{1}-733,704^{\mathrm{d}}, 627^{\mathrm{c}}$ \\
& & $\mathrm{B}_{2}-724,720^{\mathrm{c}}, 627^{\mathrm{c}}$ \\
& & \\
\hline
\end{tabular}

a Parallel pleated sheet.

${ }^{b}$ Parallel rippled sheet.

${ }^{c}$ Contribution of $\mathrm{NH}$ ob $>10$ to $\mathrm{PED}$, but no $\mathrm{CN} t$ contribution.

${ }^{\mathrm{d}} \mathrm{CN} \mathrm{t}(58)$, NH ob(8).

The dependence of the amide I frequency on the intersheet separation is shown in Fig. 2. It is clear from this figure that, in a multisheet structure, the DDC varies significantly with the sheet arrangement and the intersheet distance. Therefore, the intersheet structure must be specified in detail before reliable amide I frequencies can be predicted. For example, for parallel sheets and an intersheet separation of $5.3 \AA$ (comparable to that in $\beta$-poly(Lalanine $)^{9}$ ), Fig. 2(a) shows that the amide I frequencies would be $1669(\mathrm{~A})$ and 1677 (B) $\mathrm{cm}^{-1}$. Thus, the ir-active mode would be at a higher frequency than the Raman-active mode, the reverse of the situation for the single sheet. For antiparallel sheets [Fig. 2(b)], these frequencies would be 1682(A) an 1668(B) $\mathrm{cm}^{-1}$. [We recall that for APPS poly(L-alanine), the strong bands are observed at $1669 \mathrm{~cm}^{-1}$ in the Raman and $1632 \mathrm{~cm}^{-1}$ in the ir. $\left.{ }^{1}\right]$

The amide II modes of a single sheet are predicted at $1553(\mathrm{~A})$ and $1586(\mathrm{~B})$ $\mathrm{cm}^{-1}$, with the A-species mode expected to be strong in the ir (amide II modes are weak in the Raman). In VGG, the strong ir band is predicted at $1541 \mathrm{~cm}^{-1}$ and observed at $1543 \mathrm{~cm}^{-1} \cdot{ }^{19}$ The frequency in the PPS will be sensitive to the hydrogen-bond geometry and insensitive to sheet packing for the parallel arrangement [Fig. 3(a)], while the sensitivity to sheet packing is somewhat greater for the antiparallel arrangement [Fig. 3(b)].

As we have already observed, ${ }^{1}$ the $\mathrm{NH}$ in-plane bend (ib) coordinate contributes to modes in the $-1400-1200-\mathrm{cm}^{-1}$ region, some of which contain CN stretch (s) and have been referred to as amide III. On this basis, only the $1243(\mathrm{~A}) \mathrm{cm}^{-1}$ mode would qualify as an amide III mode, although there is a 


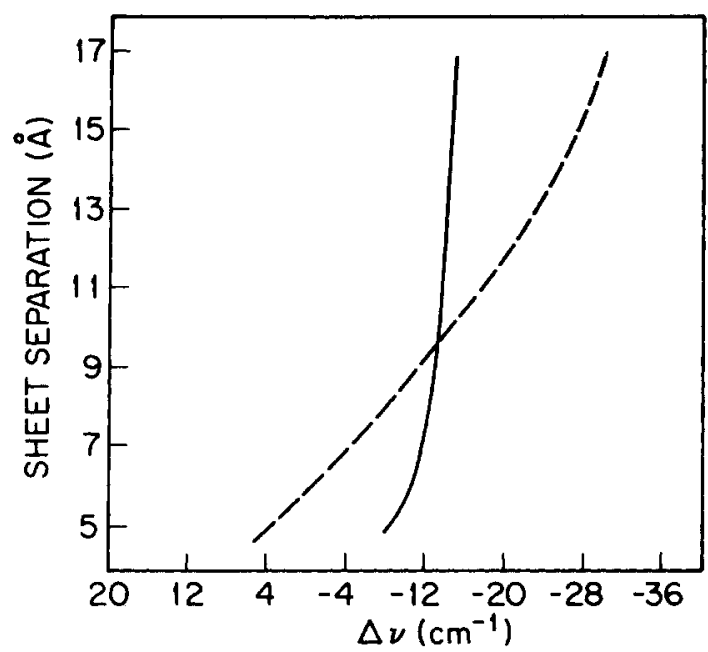

(a)

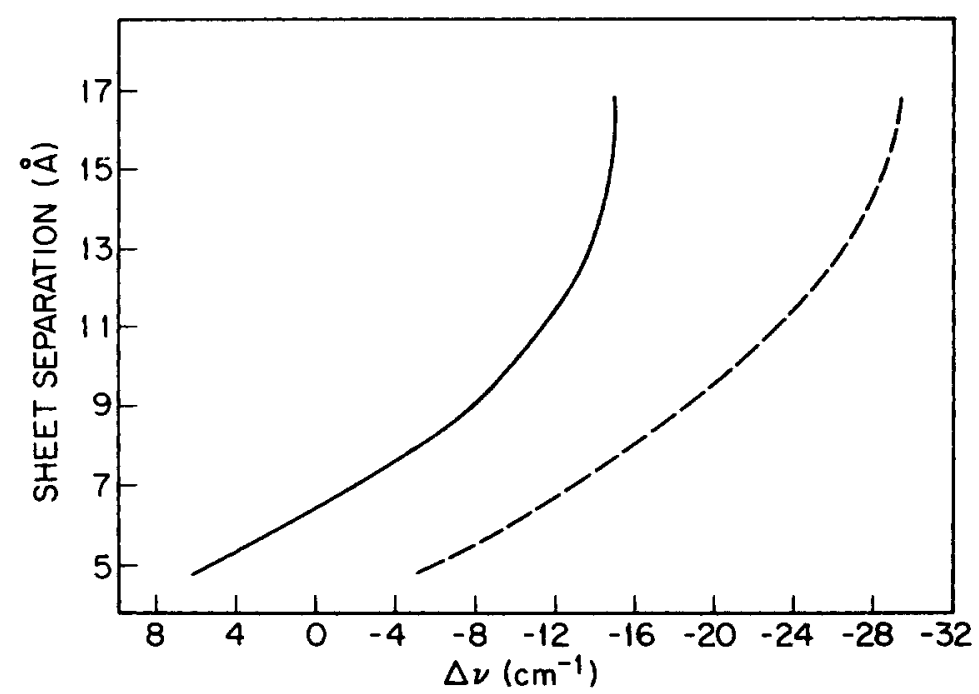

(b)

Fig. 2. Dependence of amide I frequency shifts (due to dipole derivative coupling) on intersheet separation for pleated-sheet poly(L-alanine): (-) A species, (--) B species. (a) Parallel-sheet arrangement, (b) antiparallel-sheet arrangement.

larger $\mathrm{NH}$ ib contribution to the $1330(\mathrm{~B}) \mathrm{cm}^{-1}$ mode and lesser ones to modes at $1399(\mathrm{~A})$ and $1207(\mathrm{~A}) \mathrm{cm}^{-1}$. [A similar situation exists for APPS poly(Lalanine), ${ }^{1}$ although the number of modes is increased because of the doubling of the number of peptide groups in the repeat unit: the equivalent calculated modes are at $1402(\mathrm{~A}), 1399\left(\mathrm{~B}_{1}\right) ; 1332\left(\mathrm{~B}_{3}\right), 1317\left(\mathrm{~B}_{2}\right) ; 1299\left(\mathrm{~B}_{2}\right), 1236(\mathrm{~A})$, $1231\left(\mathrm{~B}_{1}\right)$; and $1198(\mathrm{~A}), 1195\left(\mathrm{~B}_{1}\right) \mathrm{cm}^{-1}$, with $\mathrm{CN} \mathrm{s}$ contributing only to the third group.] It is interesting that $\mathrm{CN}$ s makes no contribution greater than 5 in the PED to such modes in VGG, which are calculated at 1389, 1267, 1260, and $1238 \mathrm{~cm}^{-1}$, with observed counterparts. ${ }^{19}$ This emphasizes the need to 


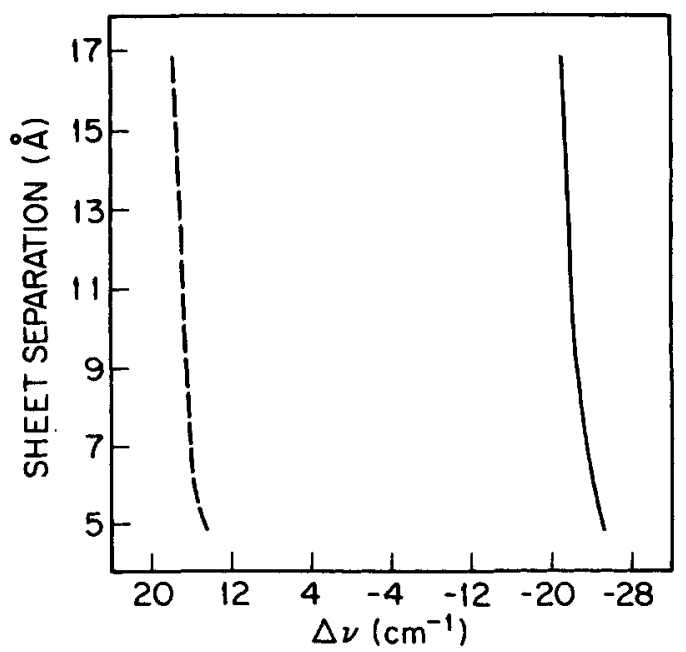

(a)

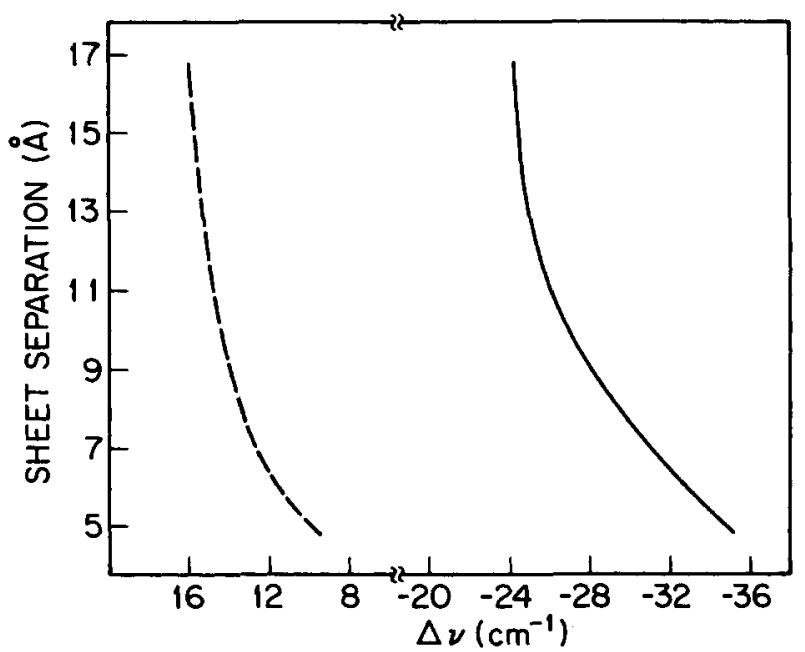

(b)

Fig. 3. Dependence of amide II frequency shifts (due to dipole derivative coupling) on intersheet separation for pleated-sheet poly(L-alanine): (-) A species, (--) B species. (a) Parallel-sheet arrangement, (b) antiparallel-sheet arrangement.

generalize our characterization of the so-called amide III mode. ${ }^{1}$

Amide V modes involve a combination of $\mathrm{NH}$ out-of-plane bend (ob) and $\mathrm{CN}$ torsion (t), and are found in the range of $\sim 750-550 \mathrm{~cm}^{-1} .{ }^{1}$ In the PPS, such modes are predicted at $712(\mathrm{~A})$ and $706(\mathrm{~B}) \mathrm{cm}^{-1}$, although a mode at 628(B) $\mathrm{cm}^{-1}$ also contains a significant contribution from $\mathrm{NH}$ ob. These frequencies are quite sensitive to the hydrogen-bond geometry, ${ }^{1}$ and therefore will depend on the specific structure of the PPS. In VGG, the amide V modes are observed (and predicted) at $714(698)$ and $675(677) \mathrm{cm}^{-1}$, with $\mathrm{NH}$ ob alone making contributions to bands at 670(662), 647(654), and $565(566) \mathrm{cm}^{-1} \cdot{ }^{19}$ In 
APPS poly(L-alanine), amide $V$ modes occur at $706(706,705)$ and $698(704)$ $\mathrm{cm}^{-1} \cdot 1$

In the case of the nonamide modes, most of the predicted frequencies are within $10 \mathrm{~cm}^{-1}$ of the average of the $A+B_{1}$ (for $A$ ) or $B_{2}+B_{3}$ (for $B$ ) frequencies of APPS poly(L-alanine). This is expected on the basis of the symmetry of the two structures and the similarity in chain conformations. However, some modes differ by $20 \mathrm{~cm}^{-1}$ or more: 643(A) in the PPS [623 in the APPS], 569(B) [592], 417(A) [440], 355(A) [328], and 296(A) [275] $\mathrm{cm}^{-1}$. Most of these have significantly different PEDs in the two structures, and it is likely that this is a reflection of the conformational differences between the two kinds of chains. This skeletal deformation region is where the effects of such small structural differences would be expected to show up, and is clearly an important region in which to look for differences between the PPS and APPS structures.

\section{PARALLEL-CHAIN RIPPLED SHEET}

As noted above, a PRS structure is feasible only for polyglycine. We present in Table $\mathrm{V}$ the calculated modes for such a single sheet. The amide modes, with DDC included for amide I and amide II, are given in Table IV.

For the single sheet, the strong Raman-active amide I mode will be the $A_{1}$ species, at $1647 \mathrm{~cm}^{-1}$, which is much lower in frequency than the strong ir-active $B_{1}$-species mode, at $1667 \mathrm{~cm}^{-1}$. This is the reverse of the situation in the APRS structure, ${ }^{1}$ but since the calculations for APRS polyglycine included resonance coupling between multiple sheets, ${ }^{10,25}$ a direct comparison of frequencies for amide I and II must be made with a multiple-sheet result. For a parallel-sheet arrangement in the polyglycine I structure ${ }^{7}$ we find: amide I $-\mathrm{A}_{1}: 1647, \mathrm{~A}_{2}: 1776, \mathrm{~B}_{1}: 1696, \mathrm{~B}_{2}: 1680$; amide $\mathrm{II}-\mathrm{A}_{1}: 1530, \mathrm{~A}_{2}: 1535, \mathrm{~B}_{1}$ : $1568, \mathrm{~B}_{2}: 1573 \mathrm{~cm}^{-1}$. For a similar antiparallel-sheet arrangement we find: amide $\mathrm{I}-\mathrm{A}_{1}: 1642, \mathrm{~A}_{2}: 1797, \mathrm{~B}_{1}: 1671, \mathrm{~B}_{2}: 1687$; amide $\mathrm{II}-\mathrm{A}_{1}: 1532, \mathrm{~A}_{2}$ : $1529, B_{1}: 1567, B_{2}: 1565 \mathrm{~cm}^{-1}$. Thus, even with intersheet coupling the strong Raman-active mode of the PRS of polyglycine would be predicted to be at a lower frequency than the strong ir-active mode. The strong ir-active amide II mode will be the $A_{1}$ species; its values of $1530-1532 \mathrm{~cm}^{-1}$ are somewhat higher than the $1517 \mathrm{~cm}^{-1}$ found (and predicted at $1515 \mathrm{~cm}^{-1}$ ) for APRS polyglycine.

The main amide III modes are calculated at similar frequencies in the two structures: $1304\left(B_{g}\right)$ and $1286\left(B_{u}\right) \mathrm{cm}^{-1}$ in the APRS structure and $1290\left(B_{1}\right)$ and $1288\left(B_{2}\right) \mathrm{cm}^{-1}$ in the PRS structure. The calculated amide $V$ modes span similar ranges: $702-736 \mathrm{~cm}^{-1}$ in the APRS and $698-733 \mathrm{~cm}^{-1}$ in the PRS structures.

Most of the nonamide modes are at comparable frequencies, but some show very large differences between the APRS and PRS, in part related to the different symmetries of the two structures $\left(C_{2 h}\right.$ for the former and $C_{2 v}$ for the latter) and in part to the different chain conformations (cf. Table I). These differences are a result of the differing eigenvectors for the normal modes. Thus, comparing the PRS and APRS [in brackets] structures, we find: $\mathrm{CH}_{2}$ rock $(r)$ at $893\left(A_{1}\right)$ and $891\left(A_{2}\right)$ [946( $\left.A_{g}\right)$ and $\left.940\left(A_{u}\right)\right] ; C O$ ob, $C O$ ib at 680 $\left(A_{1}\right)$ and $676\left(A_{2}\right)\left[630\left(A_{g}\right)\right.$ and $\left.629\left(A_{u}\right)\right] ; C^{\alpha} \mathrm{CN}$ deformation $(d)$ at $540\left(B_{1}\right)$ 
TABLE V

Calculated Normal Modes of a Parallel-Chain Rippled Sheet of Polyglycine

\begin{tabular}{|c|c|c|c|c|}
\hline \multicolumn{4}{|c|}{ Species } & \multirow[b]{2}{*}{ Potential Energy Distribution } \\
\hline$A_{1}$ & $\mathbf{A}_{2}$ & $\mathrm{~B}_{1}$ & $\overline{B_{2}}$ & \\
\hline 3284 & 3284 & 3284 & 3284 & $\mathrm{NH} s(98)$ \\
\hline 2930 & 2930 & 2931 & 2931 & $\mathrm{CH}_{2}$ as $(99)$ \\
\hline 2860 & 2860 & 2860 & 2860 & $\mathrm{CH}_{2} \mathrm{ss}(99)$ \\
\hline 1675 & 1676 & 1677 & 1676 & $\mathrm{COs}(75) \mathrm{CN} s(19) \mathrm{C}^{\alpha} \mathrm{CN} d(11)$ \\
\hline \multirow[t]{2}{*}{1543} & 1541 & & & $\mathrm{NH} \mathrm{ib}(51) \mathrm{CN} \mathrm{s}(24) \mathrm{CO} \mathrm{ib}(11) \mathrm{C}^{\alpha} \mathrm{Cs}(11)$ \\
\hline & & 1551 & 1552 & 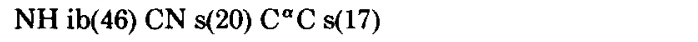 \\
\hline \multirow[t]{2}{*}{1452} & 1452 & & & $\mathrm{CH}_{2} \mathrm{~b}(82)$ \\
\hline & & 1442 & 1442 & $\mathrm{CH}_{2} \mathrm{~b}(92)$ \\
\hline \multirow[t]{3}{*}{1405} & 1405 & & & $\mathrm{CH}_{2} \mathrm{w}(47) \mathrm{CH}_{2} \mathrm{~b}(15) \mathrm{NH} \mathrm{ib}(14) \mathrm{C}^{\alpha} \mathrm{C} \mathrm{s}(13)$ \\
\hline & & 1338 & 1338 & $\mathrm{CH}_{2} \mathrm{w}(88)$ \\
\hline & & 1290 & 1288 & $\mathrm{NH} i b(44) \mathrm{C}^{\alpha} \mathrm{C} s(16) \mathrm{CO} \mathrm{ib}(14) \mathrm{CN} \mathrm{s}(10)$ \\
\hline \multirow[t]{2}{*}{1255} & 1255 & & & $\mathrm{CH}_{2} \mathrm{tw}(57) \mathrm{CH}_{2} \mathrm{w}(30)$ \\
\hline & & 1224 & 1225 & $\mathrm{CH}_{2} \mathrm{tw}(86)$ \\
\hline 1218 & 1219 & & & $\mathrm{CH}_{2} \mathrm{tw}(34) \mathrm{NH} \mathrm{ib}(19) \mathrm{CN} s(17) \mathrm{CH}_{2} \mathrm{w}(17) \mathrm{NC}^{\alpha} \mathrm{s}(14)$ \\
\hline \multirow[t]{2}{*}{1139} & 1138 & & & $\mathrm{NC}^{\alpha} \mathrm{s}(66) \mathrm{C}^{\alpha} \mathrm{Cs}(15) \mathrm{NH} \mathrm{ib}(5)$ \\
\hline & & 1029 & 1030 & $\mathrm{NC}^{\alpha} \mathrm{s}(67) \mathrm{CH}_{2} \mathrm{r}(17) \mathrm{CH}_{2} \mathrm{w}(10)$ \\
\hline \multirow[t]{2}{*}{1009} & 1004 & & & $\mathrm{CH}_{2} \mathrm{r}(38) \mathrm{CO} \mathrm{s}(15) \mathrm{C}^{\alpha} \mathrm{C} \mathrm{s}(13)$ \\
\hline & & 986 & 988 & $\mathrm{CH}_{2} \mathrm{r}(44) \mathrm{CN} \mathrm{s}(21) \mathrm{NC}^{\alpha} \mathrm{s}(15) \mathrm{CNC}^{\alpha} \mathrm{d}(11)$ \\
\hline \multirow[t]{6}{*}{893} & 891 & & & $\mathrm{CH}_{2} \mathbf{r}(26) \mathrm{CN} \mathrm{s}(16) \mathrm{NC}^{\alpha} \mathrm{C} \mathrm{d}(16) \mathrm{CO} o b(13) \mathrm{NH} \mathrm{ob}(6)$ \\
\hline & & $\begin{array}{l}879 \\
733\end{array}$ & 886 & $\begin{array}{l}\mathrm{C}^{\alpha} \mathrm{Cs} s(34) \mathrm{CN} s(19) \mathrm{CO} \mathrm{s}(13) \mathrm{CH}_{2} \mathrm{r}(11) \mathrm{C}^{\alpha} \mathrm{CN} \mathrm{d}(10) \\
\mathrm{CN} \mathrm{t}(24) \mathrm{NH} \text { ob(18) } \mathrm{NH} \ldots \mathrm{Ob}(15) \mathrm{CO} \mathrm{ib}(14) \mathrm{C}^{\alpha} \mathrm{Cs}(13)\end{array}$ \\
\hline & & & 724 & $\mathrm{CN} \mathrm{t}(68) \mathrm{NH} \ldots \mathrm{O} b(22) \mathrm{NH} \mathrm{ob}(12)$ \\
\hline & & & 720 & $\begin{array}{l}\mathrm{CO} i b(19) \mathrm{C}^{\alpha} \mathrm{C} s(15) \mathrm{NH} \text { ob(12) } \mathrm{NC}^{\alpha} \mathrm{Cd}(11) \mathrm{NC}^{\alpha} \mathrm{s}(11) \\
\mathrm{CN} \mathrm{t}(11)\end{array}$ \\
\hline & & 704 & & $\mathrm{CN} t(58) \mathrm{NH} . . . \mathrm{O} b(16) \mathrm{CO} \mathrm{ib}(10) \mathrm{NH} \mathrm{ob}(8)$ \\
\hline & 703 & & & $\mathrm{CN} t(69) \mathrm{NH} \ldots \mathrm{O} b(28) \mathrm{NH}$ ob(16) \\
\hline 698 & & & & CN t(81) NH ... O b(31) NH ob(21) \\
\hline \multirow[t]{3}{*}{680} & & & & $\begin{array}{l}\mathrm{CO} \text { ob(25) } \mathrm{CO} \mathrm{ib}(18) \mathrm{CH}_{2} \mathrm{r}(16) \mathrm{NH} \text { ob(11) } \mathrm{NC}^{\alpha} \mathrm{C} d(10) \\
\mathrm{NH} \mathrm{ib(5)}\end{array}$ \\
\hline & 676 & & & $\mathrm{CO} \mathrm{ob}(25) \mathrm{CH}_{2} \mathrm{r}(18) \mathrm{NH} \mathrm{ob}(17) \mathrm{CO}$ ib(12) \\
\hline & & 627 & 627 & $\mathrm{CO}$ ob(82) $\mathrm{NH}$ ob(16) $\mathrm{NC}^{\alpha} \mathrm{C} d(12)$ \\
\hline \multirow[t]{2}{*}{578} & 576 & & & $\mathrm{CO}$ ib(42) $\mathrm{CO} o b(28) \mathrm{C}^{\alpha} \mathrm{Cs}(19)$ \\
\hline & & 540 & 541 & $\mathrm{C}^{a} \mathrm{CN} d(49) \mathrm{NC}^{\alpha} \mathrm{C} d(12) \mathrm{NH} o b(7)$ \\
\hline \multirow[t]{4}{*}{393} & 390 & & & $\mathrm{C}^{\alpha} \mathrm{CN} d(44) \mathrm{NC}^{\alpha} \mathrm{s}(12) \mathrm{NC}^{\alpha} \mathrm{C} \mathrm{d}(11)$ \\
\hline & & 382 & 380 & $\mathrm{NC}^{\alpha} \mathrm{C} d(28) \mathrm{C}^{\alpha} \mathrm{CN} d(15) \mathrm{CO} \mathrm{ib}(13) \mathrm{NH}$ ob(8) \\
\hline & & 267 & 255 & $\mathrm{CNC}^{\alpha} \mathrm{d}(55) \mathrm{CO} \mathrm{ib}(28)$ \\
\hline & 251 & & & $\mathrm{CNC}^{\alpha} \mathrm{d}(40) \mathrm{H} \ldots \mathrm{O} \mathrm{s}(16) \mathrm{NH}$ ob(8) \\
\hline \multirow[t]{2}{*}{232} & & & & $\mathrm{CNC}^{\alpha} \mathrm{d}(30) \mathrm{NH}$ ob(18) $\mathrm{CO}$ ob(15) \\
\hline & 201 & & & $\mathrm{NH}$ ob(30) CO ob(19) CNC ${ }^{\alpha} d(12) \mathrm{C}^{\alpha} \mathrm{CN} d(10)$ \\
\hline 189 & & & & $\mathrm{CNC}^{\alpha} \mathrm{d}(38) \mathrm{C}^{\alpha} \mathrm{CN} \mathrm{d}(24) \mathrm{NH}$ ob(20) $\mathrm{CO}$ ob(10) \\
\hline \multirow[t]{7}{*}{151} & & & & $\begin{array}{l}\mathrm{NC}^{\alpha} \mathrm{C} d(24) \mathrm{NH} \text { ob(19) NH... O b(12) CN t(11) } \\
\mathrm{H} \ldots \mathrm{O} s(10)\end{array}$ \\
\hline & 138 & & & $\mathrm{NC}^{\alpha} \mathrm{C} d(33) \mathrm{NH} \ldots \mathrm{O} b(16) \mathrm{CN} t(15) \mathrm{NH}$ ob(10) \\
\hline & & 117 & & $\mathrm{H} \ldots \mathrm{Os}(87)$ \\
\hline & & 94 & & $\begin{array}{l}\mathrm{CN} \mathrm{t(34)} \mathrm{NH} \mathrm{ob}(27) \mathrm{CO} \ldots \mathrm{H} \mathrm{t}(23) \mathrm{C}^{\alpha} \mathrm{C} \mathrm{t}(17) \\
\mathrm{CO} \ldots \mathrm{H} \mathrm{b}(12)\end{array}$ \\
\hline & & & 82 & $\begin{array}{l}\mathrm{CN} t(38) \mathrm{NH} \text { ob(30) } \mathrm{NH} \ldots \mathrm{O} b(22) \mathrm{C}^{\alpha} \mathrm{C} \mathrm{t}(22) \\
\mathrm{NC}^{\alpha} \mathrm{t}(11) \mathrm{CO} \ldots \mathrm{H} \mathrm{b}(10)\end{array}$ \\
\hline & 76 & & & $\mathrm{CO} \ldots \mathrm{H} \mathrm{t}(56) \mathrm{NH}$ ob(36) CN t(23) $\mathrm{NC}^{\alpha} \mathrm{C} \mathrm{d}(12)$ \\
\hline & 39 & & & $\mathrm{H} \ldots \mathrm{O} s(36) \mathrm{CO} \ldots \mathrm{Hb}(30) \mathrm{NH} \ldots \mathrm{O} t(12) \mathrm{NH}$ ob(9) \\
\hline \multirow[t]{2}{*}{39} & & & & $\mathrm{CO} \ldots \mathrm{H} \mathrm{t}(62) \mathrm{H} \ldots \mathrm{Os}(14) \mathrm{NH} \ldots \mathrm{Ob}(13)$ \\
\hline & & & 39 & $\mathrm{CO} \ldots \mathrm{H} \mathrm{t}(58) \mathrm{CN} \mathrm{t}(20) \mathrm{NH}$ ob(6) \\
\hline
\end{tabular}

${ }^{a}$ s, Stretch; as, antisymmetric stretch; ss, symmetric stretch; b, angle bend; ib, in-plane angle bend; ob, out-of-plane angle bend; $w$, wag; tw, twist; $r$, rock; $t$, torsion; $d$, deformation. Only contributions of 10 or greater are included, except for $\mathrm{NH}$ modes, where contributions $>5$ are included. If differences in PED are $\leq 4$, modes are not listed separately and average PED is given. 
and 541( $\left.\mathrm{B}_{2}\right)\left[587\left(\mathrm{~B}_{\mathrm{g}}\right)\right.$ and $\left.589\left(\mathrm{~B}_{\mathrm{u}}\right)\right] ; \mathrm{C}^{\alpha} \mathrm{CN} \mathrm{d}$ at 393( $\left.\mathrm{A}_{1}\right)$ and $390\left(\mathrm{~A}_{2}\right)\left[291\left(\mathrm{~A}_{\mathrm{g}}\right)\right.$ and $\left.290\left(\mathrm{~A}_{\mathrm{u}}\right)\right]$; and $\mathrm{NC}^{\alpha} \mathrm{C} \mathrm{d}$ at $382\left(\mathrm{~B}_{1}\right)$ and $380\left(\mathrm{~B}_{2}\right) \mathrm{cm}^{-1}\left[323\left(\mathrm{~B}_{\mathrm{g}}\right)\right.$ and $320\left(\mathrm{~B}_{\mathrm{u}}\right)$ $\left.\mathrm{cm}^{-1}\right]$. In one case, a complex skeletal mode calculated at $768\left(\mathrm{~B}_{\mathrm{g}}\right)$ and $767\left(\mathrm{~B}_{\mathrm{u}}\right)$ $\mathrm{cm}^{-1}$ in the APRS has no counterpart in the PRS structure. As with the pleated-sheet structure, the skeletal deformation region is a sensitive probe of polypeptide chain conformation.

\section{CONCLUSIONS}

Normal mode calculations of parallel-chain pleated and rippled sheet structures show that their vibrational frequencies are different from their antiparallel-chain counterparts. In all cases there are differences in amide I and II modes, primarily due to differences in resonance coupling effects, and in some of the skeletal deformation modes. The amide I and II mode frequencies are particularly sensitive to sheet arrangement and separation, which determine the magnitudes of the shifts due to DDC. The differences in the skeletal deformation region are due to differences in chain conformations and in symmetries of the structures.

An important conclusion that emerges from these, as well as our earlier, ${ }^{1,19}$ studies is that it is important to have both ir and Raman spectra in order to make a definitive assignment of structure, particularly from the amide I region. Thus, Toniolo and Palumbo ${ }^{26}$ have proposed that homoheptamers of valine, isoleucine, and phenylalanine form parallel-chain $\beta$-sheet structures in the solid state, based on an ir amide I band at $1635-1639 \mathrm{~cm}^{-1}$ accompanied by the absence of a band in the $1690-1700-\mathrm{cm}^{-1}$ region (associated with the APPS structure). While this is an interesting suggestion, particularly in view of our calculated single-sheet amide I frequency of $1642 \mathrm{~cm}^{-1}$, it cannot be considered definitive in the absence of additional data, especially from the Raman spectrum. As we have seen, the relative ir and Raman frequencies depend strongly on the intersheet arrangement and separation, and thus together they can provide strong indicators of the structure. It would also be important to have data in the skeletal deformation region.

The studies reported here should provide greater insight into the characteristic vibrational modes of parallel-chain $\beta$-sheet structures.

This research was supported by NSF grants DMB-8517812 and DMR-8303610.

\section{References}

1. Krimm, S. \& Bandekar, J. (1986) Adv. Protein Chem. 38, 181-364.

2. Tasumi, M., Takeuchi, H., Ataka, S., Dwivedi, A. M. \& Krimm, S. (1982) Biopolymers 21, $711-714$.

3. Dwivedi, A. M. \& Krimm, S. (1982) Macromolecules 15, 186-193.

4. Dwivedi, A. M. \& Krimm, S. (1983) Macromolecules 16, 340.

5. Pauling, L. \& Corey, R. B. (1951) Proc. Natl. Acad. Sci. USA 37, 235-240.

6. Pauling, L. \& Corey, R. B. (1953) Proc. Natl. Acad. Sci. USA 39, 253-256.

7. Lotz, B. (1974) J. Mol. Biol. 87, 169-180.

8. Colonna-Cesari, F., Premilat, S. \& Lotz, B. (1974) J. Mol. Biol. 87, 181-190.

9. Amott, S., Dover, S. D. \& Elliott, A. (1967) J. Mol. Biol. 30, 201-208.

10. Dwivedi, A. M. \& Krimm, S. (1982) Macromolecules 15, 177-185.

11. Rossmann, M. G., Adams, M. J., Buehner, M., Ford, G. C. Kackert, M. L., Lentz, P. J., McPherson, A., Schevitz, R. W. \& Smiley, I. E. (1972) Cold Spring Harbor Symp. Quant. Biol. 36, 179-191. 
12. Banner, D. W., Bloomer, A. C., Petsko, G. A., Phillips, D. C., Pogson, C. I. \& Wilson, I. A. (1975) Nature 255, 609-614.

13. Quiocho, F. A. \& Lipscomb, W. N. (1971) Adv. Protein Chem. 25, 1-78.

14. Wright, C. S., Alden, R. A. \& Kraut, J. (1969) Nature 221, 235-242.

15. Ploegman, J. H. (1977) Ph.D. Dissertation, University of Groningen, Groningen, The Netherlands.

16. Salemme, F. R. (1983) Progr. Biophys. Mol. Biol. 42, 95-133.

17. Ashida, T., Tanaka, I. \& Yamane, T. (1981) Int. J. Peptide Protein Res. 1, 322-329.

18. Lalitha, V., Murali, R. \& Subramanian, E. (1986) Int. J. Peptide Protein Res. 27, 472-477.

19. Bandekar, J. \& Krimm, S. (1988) Biopolymers 27, 885-908.

20. Cheam, T. C. \& Krimm, S. (1984) Chem. Phys. Lett. 107, 613-616.

21. Cheam, T. C. \& Krimm, S. (1985) J. Chem. Phys. 82, 1631-1641.

22. Moore, W. H. \& Krimm, S. (1975) Proc. Natl. Acad. Sci. USA 72, 4933-4935.

23. Moore, W. H. \& Krimm, S. (1976) Biopolymers 15, 2465-2483.

24. Chirgadze, Yu. N. \& Nevskaya, N. A. (1976) Biopolymers 15, 627-636.

25. Moore, W. H. \& Krimm, S. (1976) Biopolymers 15, 2439-2464.

26. Toniolo, C. \& Palumbo, M. (1977) Biopolymers 16, 219-224.

Received July 8, 1987

Accepted December 10, 1987 